\title{
EDUCAÇÃO EMPREENDEDORA EM UMA PLATAFORMA TECNOLÓGICA LUDIFICADA: UM ESTUDO DE CASO
}

\author{
Hudson Costa - SEBRAE - hudsonrc@hotmail.com
}

Edilson Ferneda - Universidade Católica de Brasília - eferneda@ pos.ucb.com Hercules Antonio do Prado - Universidade Católica de Brasília - hercules@ucb.br

\begin{abstract}
Resumo. Os processos de aprendizagem enfatizam cada vez mais a compreensão, independência, empoderamento e melhoria das habilidades dos estudantes, nascidos e imersos na cultura digital. O uso de jogos para aumentar o interesse dos alunos em atividades de ensino e aprendizagem não é recente, mas, principalmente no contexto da cultura digital, extrapolar seu aspecto lúdico continua sendo um desafio. Este trabalho discute a aplicação de elementos e da mecânica de jogos em uma plataforma tecnológica no campo da educação empresarial. Foi realizado um estudo de caso com uma plataforma de aprendizado ludificada para verificar sua adequação no desenvolvimento do comportamento empreendedor em seus usuários. A plataforma foi analisada de acordo com o modelo de gamificação Octalysis e mostrou-se limitada quanto ao desenvolvimento de competências empresariais. Contudo, verificou -se que a gamificação aplicada à educação empresarial tem potencial como alternativa para melhorar o engajamento e outras características esperadas em empreend edores. Neste sentido, foi proposto um conjunto de melhorias para tornar a plataforma mais aderente às dimensões preconizadas pelo Octalysis.
\end{abstract}

Palavras-chave: Educação Empreendedora, Gamificação, Motivação.

\section{ENTREPRENEURSHIP EDUCATION IN A GAMIFIED TECHNOLO GICAL PLATFORM: A CASE STUDY}

\begin{abstract}
Learning processes increasingly emphasize the understanding, independence, empowerment and improvement of students' abilities, born and immersed in the digital culture. The use of games to increase students' interest in teaching and learning activities is not new. However, overcoming the role of game as entertainment, mainly in the context of digital culture, remains a challenge. This paper discusses the application of elements and game mechanics in a technological platform in the field of business education. The case study was carried out with a ludified learning platform to verify its suitability in the development of the entrepreneurial behavior in its users. The platform was analyzed according to the Octalysis model for gamification and shown to be limited in the development of entrepreneurial skills. However, it has been found that gamification applied to entrepreneurial education has potential to improve engagement and other characteristics expected in entrepreneurs. In this sense, it was proposed a set of improvements to make the platform more adherent to the dimensions advocated by Octalysis.
\end{abstract}

Keywords: Entrepreneurial education, Gamification, Motivation.

\section{INTRODUÇÃO}

Os processos de aprendizagem vêm sofrendo transformações para uma nova forma baseada na compreensão, independência, capacitação e melhoria de competências dos estudantes. O ensino baseado em jogos é um exemplo dessas novas formas de aprendizagem. $\mathrm{O}$ ato de jogar, além de proporcionar prazer, é um meio para se desenvolver habilidades cognitivas, estimulando a atenção e a memória (Furió et al., 
2013). O uso de elementos de jogos para suscitar nos estudantes maior engajamento e satisfação com o processo de aprendizado não é uma ideia recente. O desafio, porém, é fazer com que seus aspectos lúdicos não ofusquem os objetivos pedagógicos.

Nesse cenário, surgem iniciativas para oferecer motivação, engajamento, prazer e diversão na transformação de uma realidade ou de um cotidiano, em uma situação lúdica, baseada na utilização de mecânicas e técnicas de jogos, com o objetivo de influenciar as pessoas para um determinado objetivo. Desta forma, tem-se a chamada gamification - gamificação, ou ainda ludificação (Deterding, 2012, Werbach e Hunter, 2012, Zichermann e Cunningham 2011).

Apesar do crescente interesse por gamification, sua abordagem no âmbito acadêmico é recente (Hamari et al., 2014; Pedersen; Poulsen, 2016). Por outro lado, diversos trabalhos apontam para a relevância dos jogos na educação (Smale et a 1. 2016; Al-Azawi et al. 2016), em particular a educação empreendedora (Mayer et al., 2013).

Avalia-se como a utilização de gamification em plataforma tecnológica de ensino contribui para o desenvolvimento das características do comportamento empreendedor (CCEs) em jovens universitários. Como forma de endosso à pesquisa, estudou -se um ambiente virtual voltado para a educação empreendedora.

\section{REVISÃO BIBLIOGRÁFICA}

\subsection{Gamification}

Gamification pode ser compreendida como a utilização de mecanismos de jogos em contextos que não são jogos (Deterding et al., 2011; Werbach e Hunter, 2012; Zichermann e Cunningham, 2011). Uma das características dessa té cnica é motivar, incentivar e orientar os usuários mediante elementos de interface com modelos que transmitem experiências equivalentes aos utilizados em jogos.

Gamification é usualmente utilizada com o objetivo de influenciar comportamentos desejáveis nos indivíduos durante a execução de atividades em processos de trabalho e no uso de sistemas de informação (Marczewski, 2013). Assim, o uso dessa técnica pode tornar a tecnologia mais envolvente, ajudando na solução de problemas e incentivando os usuários a interagir com determinados comportamentos do sistema, devido à prédisposição psicológica dos seres humanos em lidar com jogos e suas mecânicas.

\subsection{O modelo de Yu-Kai Chou}

Yu-kai Chou, um dos pioneiros em gamification, desde 2003 atua em pesquisas e consultorias relacionados ao assunto. Está classificado entre os maiores influenciadores na área, como pode ser constatado no portal Gamification Gurus leaderboard (https://www.leaderboarded.com/gurus). Este não é um portal científico e também não existe uma supervisão ou auditoria de um ranking, porém tem sido utilizado pelo mercado como uma fonte de consulta por ser um espaço de articulação daqueles com influência e impacto significativos relacionados à gamification. Nesse portal, as regras de pontuação para a definição dos líderes seguem os critérios: número de outros "gurus" que o seguem, retweets para \#gamification, menções no Tweeter, citações no Google+, visualizações no YouTube, visualizações no Slideshare, uploads no Instagram, uploads no Klout e pageviews no blog.

Do modelo de Chou (2014) para gamification, chamado Octalysis, constam oito "dimensões" ou unidades de núcleo: significado, fortalecimento, influências sociais, imprevisibilidade, prevenção, escassez, propriedade e realização. Na Figura 1 é apresentado o modelo Octalysis e as oito unidades de núcleos que determinam as ações e motivações dos jogadores. 


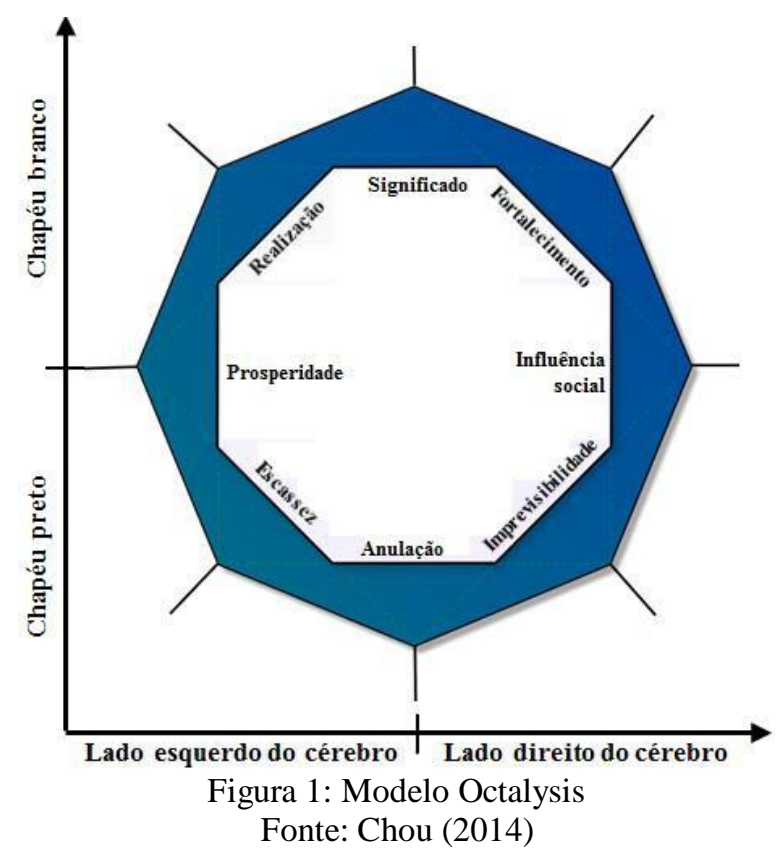

Cada unidade pode ser assim descrita: (i) Significado representa a importância para o jogador dos fatores que o motivam em relação a seus interesses pessoais; (ii) Fortalecimento representa os tipos de usuários envolvidos em um processo criativo, onde é exposto, de forma repentina, a novos fatos, que tenta combinar em diferentes situações; (iii) Influência social incorpora todos os elementos sociais que levam as pessoas a obter influência, incluindo: tutoria, aceitação, respostas sociais, companheirismo, bem como a competição e inveja; (iv) Imprevisibilidade ou curiosidade estimula no indivíduo o desejo de descobrir o que vai acontecer, fazendo com que pense recorrentemente sobre a dinâmica do jogo; (v) Anulação provoca no individuo a sensação de que as oportunidades que estão desaparecendo podem não ser mais recuperadas (em outras palavras, as pessoas sentem que se não agirem de imediato, perderão uma oportunidade); (vi) Escassez reflete o desejo de se obter algo coisa inacessível. O fato de que as pessoas não podem obter alguma coisa neste exato momento pode motivá-las a pensar sobre isso o dia todo; (vii) Propriedade é aonde os usuários são motivados a possuírem algo, e representa o núcleo para acumular riquezas, lidando com muitos bens ou moedas virtuais; (viii) Realização impulsiona o progresso, o desenvolvimento de habilidades e, eventualmente, a superação de desafios, associados, aqui, à conquista de um emblema ou troféu, por exemplo. Esta é também a unidade central que permite projetar pontos, emblemas e rankings.

Para melhor entendimento e visualização das informações em seu modelo, Chou (2014) considera que existem fatores motivacionais que são tratados em diferentes lados do cérebro. Em nota, o autor comenta que esta relação não está diretamente associada com a ciência do cérebro ou estudos de neurociência, sendo utilizado meramente como forma ilustrativa e facilidade de compreensão.

De acordo com o autor, as unidades centrais posicionadas no lado direito no diagrama Octalysis estão relacionados com a criatividade, a auto expressão e aspectos sociais, ou seja, motivações intrínsecas. Os núcleos do lado esquerdo estão associados à lógica e cálculos, ou motivações extrínsecas.

A recomendação de Chou (2014) é que ao realizar um planejamento das dinâmicas e mecânicas dos jogos, seja explorado mais o lado dir eito do cérebro. O autor considera que as motivações intrínsecas proporcionam melhores diversões e gratificação ao usuário, além de promover as motivações de forma continuada e de longo prazo. Ele 
chama 'chapéu branco' a unidade superior da imagem e 'chapéu preto' a unidade inferior. As motivações positivas (realização, significado e fortalecimento) estão localizadas na área superior do modelo, enquanto que as áreas inferiores estão localizadas as motivações negativas (escassez, prevenção e imprevisibilidad e). Os fatores relacionados ao chapéu preto permitem a obtenção de resultados produtivos de forma maliciosa ou manipuladoras. Por exemplo, existem pessoas que buscam aplicativos que trabalham com estas motivações para realizar exercícios físicos com mais frequência ou comer de forma saudável, ou seja, os aplicativos influenciam a vida do indivíduo. No estudo de caso realizado neste trabalho, descrito na seção 4, a ferramenta Octalysis foi utilizada na elaboração do modelo referente à configuração atual da plataforma, bem como para a elaboração do modelo correspondente às melhorias propostas.

\subsection{Gamification e a educação empreendedora}

De acordo com Zheng e Yang (2011), educação empreendedora pode ser entendida como um processo que proporciona a aquisição de conhecimentos e o desenvolvimento de habilidades, possibilitando ao indivíduo a identificação de oportunidades que outros não enxergariam. Segundo Heinonen e Poikkijoki (2006), seu principal objetivo é integrar habilidades e atributos de um indivíduo empreendedor com o processo empreendedor. Para Zheng e Yang (2011), a educação empreendedora, além de desenvolver conhecimento e habilidade para abertura de negócios, deve prover habilidade para pensar de forma empreendedora por meio de uma nova abordagem de ensino, com inovação e flexibilidade, considerando, inclusive, diferentes ambientes.

Por vários anos a educação empreendedora seguiu alguns estilos de ensino, às vezes de maneira tradicional e outras de forma mais inovadora (Lane, 2011; Nab et al., 2010). Alguns autores declaram que o empreendedorismo não pode ser aprendido em livros ou cursos, mas somente na prática com erros e acertos (Gstraunthaler e H endry, 2011; Nab et al., 2010; Vincett e Farlow, 2008), o que representa uma oportunidade para a utilização de jogos na simulação de situações práticas.

\subsection{Características do comportamento empreendedor (CCE)}

Comportamento empreendedor é um conceito que não se restringe à criação de negócios, mas também considera características comportamentais que podem favorecer o sucesso destas empresas. McClelland (1987) identifica dez características empreendedoras, agrupadas em três categorias (realização, planejamento e resolução de problemas e influência na relação com as pessoas), conforme apresentadas no Quadro 1.

Quadro 1: Características do comportamento empreendedor.

\section{CATEGORIA: 1.0 REALIZAÇÃO}

CCE: 1.1 Busca de oportunidades e iniciativa: Faz as coisas antes de solicitado ou antes de forçado pelas circunstâncias. Age para expandir o negócio a novas áreas, produtos ou serviços. Aproveita oportunidades fora do comum para começar um negócio, obter financiamentos, equipamentos, terrenos, local de trabalho ou assistência. CCE: 1.2 Exigência de qualidade e eficiência: Encontra maneiras de fazer as coisas melhor, mais rápido ou mais barato. Age de maneira a fazer coisas que satisfazem ou excedem padrões de excelência. Desenvolve ou utiliza procedimentos para assegurar que o trabalho seja terminado a tempo ou que o trabalho atenda a padrões de qualidade previamente combinados.

CCE: 1.3 Persistência: Age diante de um obstáculo significativo. Age repetidamente ou muda de estratégia, a fim de enfrentar um desafio ou superar um obstáculo. Faz um sacrifício pessoal ou desenvolve um esforço extraordinário para completar uma tarefa.

CCE: 1.4 Independência e autoconfiança: Busca autonomia em relação a normas e controles de outros. Mantém seu ponto de vista mesmo diante da oposição ou de resultados inicialmente desanimadores. Expressa confiança na sua própria capacidade de completar uma tarefa difícil ou de enfrentar um desafio.

CATEGORIA: 2.0 PLANEJAMENTO E RESOLUÇÃO DE PROBLEMAS

CCE: 2.1 Correr riscos calculados: Avalia alternativas e calcula riscos deliberadamente. Age para reduzir os 
riscos ou controlar os resultados. Coloca-se em situações que implicam desafios ou riscos moderados. CCE: 2.2 Busca de informações: Dedica-se pessoalmente a obter informações de clientes, fornecedores e concorrentes. Investiga pessoalmente como fabricar um produto ou fornecer um serviço. Consulta especialistas para obter assessoria técnica ou comercial.

CCE: 2.3 Estabelecimento de metas: Estabelece metas e objetivos que são desafiantes e que têm significado pessoal. Define metas de longo prazo, claras e específicas. Estabelece objetivos mensuráveis e de curto prazo.

CCE: 2.4 Planejamento e monitoramento sistemáticos: Planeja dividindo tarefas de grande porte em sub tarefas com prazos definidos. Constantemente revisa seus planos, levando em conta os resultados obtidos e mudanças circunstanciais. Mantém registros financeiros e utiliza-os para tomar decisões.

\section{CATEGORIA: 3.0 INFLUÊNCIA (RELAÇÃO COM AS PESSOAS)}

CCE: 3.1 Comprometimento: Assume responsabilidade pessoal pelo desempenho necessário ao atingimento de metas e objetivos. Colabora com os empregados ou se coloca no lugar deles, se necessário, para terminar um trabalho. Esmera-se em manter os clientes satisfeitos e coloca em primeiro lugar a boa vontade em longo prazo, acima do lucro em curto prazo.

CCE: 3.2 Persuasão e redes de contato: Utiliza estratégias deliberadas para influenciar ou persuadir os outros. Utiliza pessoas chave como agentes para atingir seus próprios objetivos. Age para desenvolver e manter relações comerciais.

Fonte: adaptado por Silva (1991) a partir de McClelland (1987)

\section{METODOLOGIA}

Realizou-se um estudo de caso aplicado a uma plataforma tecnológica de educação empreendedora que utiliza ludificação como parte de sua abordagem pedagógica que inclui 21 jogos voltados ao desenvolvimento das CCEs. Sobre essa plataforma foi adotado o seguinte percurso metodológico: (i) utilizando o modelo Octalisys, foram analisadas as principais funcionalidades da plataforma considerada e verificados os seus níveis de cobertura das CCEs; (ii) via grupo focal, foram coletadas e analisadas as percepções dos coordenadores que trabalham diretamente com a plataforma para corroborar os resultados da primeira fase; (iii) baseado nos resultados das duas primeiras etapas, foram identificados potenciais pontos de melhoria na plataforma em questão, como também apresentado um novo modelo Octalisys; (iv) por fim, comparouse a situação atual com a situação proposta. Foi realizada uma avaliação ex ante dos impactos da proposta com a atribuição de pontos às CCEs com e sem as melhorias. Tal avaliação, embora subjetiva, foi elaborada a partir de características observadas e discutidas com o grupo focal.

\section{ESTUDO DE CASO}

Neste trabalho, foi realizado um estudo de caso sobre a plataforma Desafio Universitário Empreendedor ( (http://desafiouniversitarioempreendedor.sebrae.com.br/), do SEBRAE. Esta plataforma suporta uma competição nacional de caráter educacional, com atividades presenciais e virtuais. Ao participar do Desafio Universitário, os participantes são estimulados a desenvolver atitudes empreendedoras e se tornam mais preparados para as situações do mercado, por meio da capacitação, aprimoramento e desenvolvimento de habilidades corporativas (SEBRAE, 2015). A plataforma é composta por um conjunto jogos. Os pontos conquistados em cada jogo são também acumulados na plataforma.

De acordo com o regulamento do Desafio, cada participante pode escolher qualquer jogo para começar. De forma automática, os pontos alimentam os rankings nos quais é possível visualizar os alunos que obtêm a melhor colocação a partir de seu desempenho.

Para identificar os níveis de cobertura da plataforma ludificada no desenvolvimento das CCEs, foi realizada uma análise exploratória dos jogos disponíveis na plataforma. Os autores desta pesquisa participaram como jogadores em 21 jogos, avaliando suas mecânicas e dinâmicas, identificando os níveis de cobertura que cada um possui sobre as dimensões de McClelland (1961) em relação às CCEs. Neste sentido, foram 
analisadas as características apresentadas no Quadro 1 nos jogos citados. Durante as análises, observou -se em que grau (total, parcial ou nenhum) os jogos apresentam dinâmicas que desenvolvem as características elencadas nos jogadores, considerando -se os seguintes cruzamentos de dados: (i) características por CCE; (ii) jogos por categoria; (iii) jogos por CCE; (iv) jogos por características; (v) características por jogo.

\subsection{Modelo Octalysis atual}

Para verificar a situação atual da plataforma, foi desenvolvido um modelo Octalysis considerando as características da plataforma como um todo. Dados os principais recursos hoje oferecidos pela plataforma, foram realizadas análises subjetivas $\mathrm{e}$ pontuados de 0 a 10 cada núcleo do octógono, onde 0 representa a ausência de elementos de gamification e 10 a presença de vários elementos (Quadro 2).

Quadro 2: Atuais recursos da plataforma associadas aos núcleos do modelo Octalysis

\begin{tabular}{|l|c|}
\hline \multicolumn{1}{|c|}{ Características atuais da plataforma por núcleo } & $\begin{array}{c}\text { Pontuação } \\
\text { atual }\end{array}$ \\
\hline Significado: Visão de empreendedorismo & {$[2]$} \\
\hline $\begin{array}{l}\text { Fortalecimento: Placar de pontos individuais. Ranking histórico. Nível na barra de } \\
\text { progresso. Pontos que faltam para se atingir o próximo nível }\end{array}$ & {$[3]$} \\
\hline $\begin{array}{l}\text { Influência Social: Funcionalidade "Rede de Amigos”. Adicionar jogadores em seu } \\
\text { círculo de contatos. Avaliar os pontos dos amigos. Conhecer os jogos em que seus } \\
\text { amigos participam }\end{array}$ & {$[5]$} \\
\hline $\begin{array}{l}\text { Imprevisibilidade ou curiosidade: Novos jogos publicados de tempos em tempos. } \\
\text { Novas conversas de negócio (palestras sobre empreendedorismo). Palestras publicadas } \\
\text { inesperadamente }\end{array}$ & {$[5]$} \\
\hline $\begin{array}{l}\text { Anulação: Eventos temporários: Cursos / Palestras empreendedoras / Workshop sobre } \\
\text { empreendedorismo }\end{array}$ & {$[2]$} \\
\hline $\begin{array}{l}\text { Escassez: Limitações de cursos por dia. Dias específicos para os eventos de negócio. } \\
\text { Pontuação máxima permitida em cada jogo }\end{array}$ & {$[5]$} \\
\hline Propriedade: Criação/edição do perfil individual do jogador & {$[2]$} \\
\hline $\begin{array}{l}\text { Realização: Prêmios: Viagens nacionais e internacionais / Mochila para notebook / } \\
\text { Smartphone / tablet / troféu }\end{array}$ & {$[7]$} \\
\hline
\end{tabular}

\subsection{Pesquisa junto aos coordenadores}

Buscou-se, junto aos Coordenadores do projeto Desafio Universitário, no SEBRAE Nacional, identificar crenças, sentimentos e opiniões em relação ao desenvolvimento das CCEs com a plataforma. Durante a aplicação do grupo focal, procurou -se criar um espaço para exposição de ideias. O grupo era composto por 4 especialistas, todos com experiência e conhecimento sobre a plataforma e os jogos em estudo. O roteiro (Quadro 3) foi elaborado considerando as informações e resultados obtidos nas fases de análise das funcionalidades da plataforma e na etapa de geração do modelo Octalysis, onde foram identificados os níveis de cobertura das CCEs pelos jogos, os níveis de desenvolvimento das CCEs nos jogadores e o nível de aderência da plataforma aos conceitos de gamification.

Quadro 3: Resultado da pesquisa com o grupo focal

\begin{tabular}{|c|c|c|c|c|c|}
\hline \multirow{2}{*}{ Afirmação } & \multicolumn{5}{|c|}{ Opinião * } \\
\hline & [1] & [2] & [3] & [4] & [5] \\
\hline $\begin{array}{l}\text { A plataforma desenvolve nos seus participantes mais motivações } \\
\text { intrínsecas (relacionada a impulso inato, atingir o prazer sem a } \\
\text { expectativa de recompensas materiais) do que extrínsecas } \\
\text { (impulsionada para o alcance de determinada recompensa ou prêmio). }\end{array}$ & 2 & 2 & - & - & - \\
\hline $\begin{array}{l}\text { A dinâmica dos jogos da plataforma desenvolve nos participantes as } \\
\text { CCEs relacionadas: }(i) \text { à colaboração com os empregados, (ii) à } \\
\text { manutenção dos clientes satisfeitos acima do lucro a curto prazo, (iii) } \\
\text { ao desenvolvimento do relacionamento com pessoas chave para }\end{array}$ & - & 1 & - & 2 & 1 \\
\hline
\end{tabular}




\begin{tabular}{|c|c|c|c|c|c|}
\hline $\begin{array}{l}\text { atingir seus próprios objetivos e (iv) a ações para o desenvolvimento e } \\
\text { manutenção de relacõos comerciais. }\end{array}$ & & & & & \\
\hline $\begin{array}{l}\text { A maioria dos jogos desenvolvem nos jogadores o comportamento } \\
\text { relacionado à definição de metas de curto, médio e longo prazo, de } \\
\text { maneira clara e específica. }\end{array}$ & 4 & - & - & - & - \\
\hline $\begin{array}{l}\text { Os jogos, em sua maioria, desenvolvem nos jogadores a preocupação } \\
\text { de um empresário sobre a gestão financeira e o fluxo de caixa de uma } \\
\text { empresa. }\end{array}$ & - & - & - & 2 & 2 \\
\hline $\begin{array}{l}\text { Os jogos da plataforma possuem uma relação entre si, desenvolvendo } \\
\text { características e objetivos claros e inter-relacionados, refletindo na } \\
\text { plataforma em geral os resultados e metas alcançadas nestes } \\
\text { objetivos. }\end{array}$ & 4 & - & - & - & - \\
\hline $\begin{array}{l}\text { A plataforma apresenta aos jogadores, de forma clara e específica, os } \\
\text { problemas e objetivos que, por meio dos jogos, se propõe a resolver } \\
\text { em termos de empreendedorismo. }\end{array}$ & 1 & - & - & 2 & 1 \\
\hline
\end{tabular}

* [1] - discordo totalmente ... [5] - concordo totalmente.

$\mathrm{Na}$ visão dos coordenadores, existem jogadores que são motivados a participar dos jogos pela possibilidade de aprender empreendedorismo por meio de um ambiente de aprendizagem virtual gratuita. Porém, de acordo com eles, a maioria dest es jogadores é motivada pelos prêmios e viagens oferecidos pela dinâmica do próprio desafio.

Os participantes tendem a concordar que a plataforma, quando aborda o tema relacionado ao empreendedorismo, por si só já apresenta estes objetivos, porém divergem quanto à clareza de informações sobre os tipos de problemas relativos a empreendedorismo que a plataforma se propõe a resolver.

As respostas dos especialistas corroboram os resultados obtidos durante as análises da plataforma nos seguintes aspectos: (i) nenhum jogo desenvolve todas as CCEs, (ii) a maioria dos jogos está voltada ao estímulo de comportamento relacionado à gestão financeira e administrativa de uma empresa e ( iii) os jogos não possuem relação entre si ou interconexões de objetivos.

\subsection{Proposta de melhorias na plataforma}

Tendo em vista as principais funcionalidades da plataforma avaliada, os níveis de desenvolvimento das CCEs, a avaliação do modelo Octalysis atual e o resultado da pesquisa realizada junto ao grupo focal, foram propostas melhorias a serem implementadas, das quais as principais são apresentadas no Quadro 4.

Quadro 4: Propostas de melhoria (Octalysis) sobre a plataforma

\begin{tabular}{|c|c|}
\hline Propostas de melhoria por núcleo & $\begin{array}{l}\text { Pontos c/ } \\
\text { melhorias }\end{array}$ \\
\hline $\begin{array}{l}\text { Significado: Destacar, de forma clara e objetiva, na plataforma, as vantagens, tipos de } \\
\text { desenvolvimento e conquistas intrínsecas e extrínsecas que os participantes e a sociedade } \\
\text { poderão obter ao participar do Desafio Universitário Empreendedor. }\end{array}$ & [8] \\
\hline $\begin{array}{l}\text { Fortalecimento: Apresentar mensagens de estímulo ou de alerta. Comparar o jogador } \\
\text { com ele mesmo, por exemplo, "Hoje, você conquistou mais prêmios do que a última vez } \\
\text { que você jogou. Parabéns!". Â medida que o competidor avance ou conquiste prêmios, } \\
\text { destacá-lo entre os demais competidores, incentivando e fortalecendo sua participação } \\
\text { no jogo. A cada nível ou etapa vencida, incentivar o jogador, incluindo a apresentação de } \\
\text { informações sobre o seu desempenho até aquele momento. }\end{array}$ & {$[6]$} \\
\hline $\begin{array}{l}\text { Influência Social: Considerando que jogar com outras pessoas é sempre melhor do que } \\
\text { jogar sozinho (Marczewski, 2013), proporcionar maior interação entre os jogadores, via } \\
\text { redes sociais, jogos multiplayer, simulando, por exemplo, a situação de concorrência de } \\
\text { negócio dos competidores no ramo de negócio. Disponibilizar jogos com ênfase em } \\
\text { simulação do relacionamento entre parceiros de negócio, fornecedores, especialistas em } \\
\text { consultoria, dirigido à dinâmica de desenvolvimento das CCEs relacionadas à influência } \\
\text { (relação com as pessoas) e busca de informações. }\end{array}$ & [9] \\
\hline Imprevisibilidade ou curiosidade: Apresentar obstáculos significativos de tal forma que & [7] \\
\hline
\end{tabular}




\begin{tabular}{|c|c|}
\hline $\begin{array}{l}\text { o jogador tenha que mudar de estratégia para alcançar os objetivos e metas estabelecidas, } \\
\text { assim como ocorre na vida real de um empreendedor. Interconectar os jogos, tendo em } \\
\text { vista os objetivos e metas gerais voltadas ao desenvolvimento das CCEs. Por exemplo, a } \\
\text { plataforma poderia, de forma imprevisível, desafiar o jogador a alcançar o prêmio } \\
\text { relacionado à CCE "persistência" através dos jogos X, Y e Z, e assim por diante. }\end{array}$ & \\
\hline $\begin{array}{l}\text { Anulação: Oferecer para aqueles que finalizassem uma determinada tarefa desafiadora } \\
\text { temporária, a oportunidade de ganhar prêmio ou a participação em um curso, congresso, } \\
\text { palestra ou chats com um especialista. }\end{array}$ & [9] \\
\hline $\begin{array}{l}\text { Escassez: Estimular a indicação de amigos na plataforma, ou seja, quando um jogador } \\
\text { entra na plataforma indicado por outro participante (professor ou outro jogador) e } \\
\text { informa que foi indicado, aquele que indicou obtêm destaque na plataforma e algumas } \\
\text { vantagens que os outros não possuem. Bonificar os jogadores que indicam participantes } \\
\text { para a plataforma, oferecendo alguns diferenciais tais como troféus/medalhas, cursos } \\
\text { diferenciados no SEBRAE de forma gratuita ou com desconto, entradas em áreas } \\
\text { restritas dos jogos, informações ou dicas que outros não possuem, ou créditos em bônus } \\
\text { ou viagens que podem ser utilizados no final do ciclo do desafio universitário. }\end{array}$ & [9] \\
\hline $\begin{array}{l}\text { Propriedade: Permitir através de configurações pré-definidas a criação de perfil com as } \\
\text { CCEs. Configurar de forma automática (baseada em um simples questionário) o perfil } \\
\text { empreendedor do jogador e associar este ao seu avatar. Direcionar o jogador para o } \\
\text { desenvolvimento das CCEs considerando o perfil previamente mapeado e fortalecer } \\
\text { outras características que o jogador já possua. Identificar através de monitoramento dos } \\
\text { perfis se existe uma correlação entre o perfil do jogador (CCE) com o seu desempenho } \\
\text { no Desafio Universitário. }\end{array}$ & [8] \\
\hline $\begin{array}{l}\text { Realização: Utilizar o conceito chamado na gamification de PBL (Points, badges and } \\
\text { leaderboards). Premiar por CCE conquistada. Modificar o layout e cores da plataforma à } \\
\text { medida que as CCEs são desenvolvidas (conquistadas). Modificar o perfil (avatar) do } \\
\text { jogador à medida que as CCEs são desenvolvidas (conquistadas). }\end{array}$ & [8] \\
\hline
\end{tabular}

\subsection{Modelo com as melhorias propostas}

Observa-se uma potencial evolução em todos os núcleos do modelo, com destaque para os itens Influência Social, Escassez e Anulação. A evolução do nú cleo Influência Social pode ser caracterizada por soluções que permitem maior conectividade. Implementadas as propostas aqui apresentadas, será possível obter maior interação e relacionamento entre os participantes da plataforma. O núcleo Escassez, por exe mplo, passa a gerar maior valor e dinâmica desde a entrada do participante no desafio até a sua efetiva participação em eventos, tais como workshop e palestras. A nova dinâmica permite oferecer benefícios aos jogadores que mais contribuem para o aumento da participação e colaboração dos envolvidos. No caso da Anulação, participar de eventos oferecidos pelo SEBRAE passa a ser uma forma de premiação e não um evento que gere pontos por participação, como se dá atualmente. Acredita -se que as mudanças propostas podem dar à plataforma maior competitividade e interação entre os participantes, porém o design e a forma como as informações são apresentadas podem influenciar o comportamento dos jogadores frente aos desafios apresentados nas dinâmicas dos jogos.

\section{DISCUSSÃO DOS RESULTADOS}

As principais indicações de melhoria estão relacionadas à utilização de mais recursos de dinâmicas e mecânicas oferecidos pela gamification. Sugere-se melhor destacar, na plataforma, o que representa participar do Desafio, apresentando -se os benefícios e conquistas que a sociedade brasileira, os alunos, os professores e a própria educação obteriam com o devido engajamento dos participantes nos jogos e nos desafios propostos. Infere-se que a aplicabilidade das mudanças propostas desenvolve as CCEs nos jogadores, quando todos os elementos e jogos estão interconectados e focados em direção a um objetivo geral comum: o desenvolvimento das características 
empreendedoras. A aplicação dos elementos de gamification como emblemas, troféus, prêmios, ranking geral, ranking por categoria, configurações e criação de perfis inteligentes, pode aumentar o engajamento dos participantes no Desafio e promover o desenvolvimento das CCEs. Na Figura 2 comparam-se os modelos Octalysis das situações atual (em vermelho) e com as melhorias propostas (em azul).

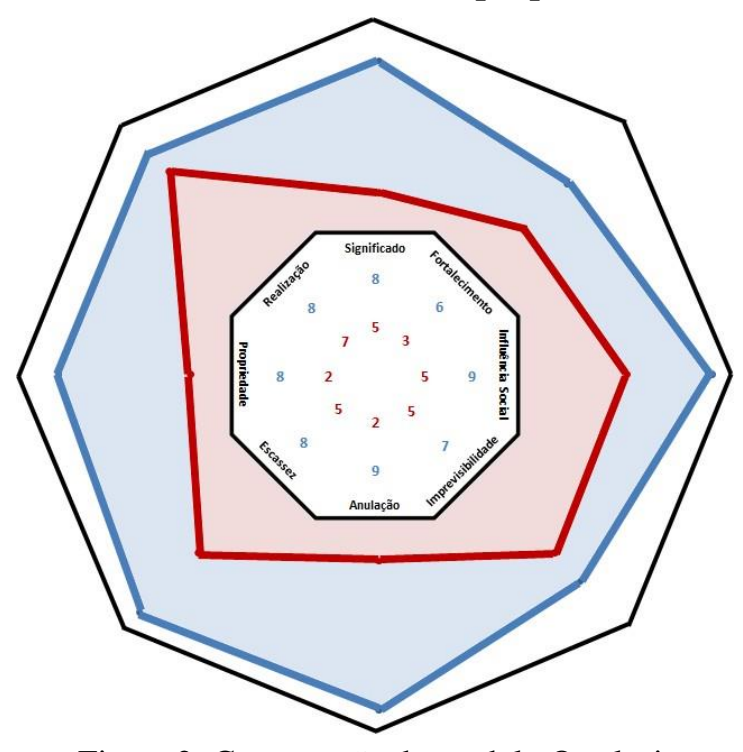

Figura 2: Comparação do modelo Octalysis

\section{CONSIDERAÇÕES FINAIS E TRABALHOS FUTUROS}

Este trabalho avaliou a influência da gamification em plataformas tecnológicas para o desenvolvimento das CCEs em jovens universitários. Foi realizado um estudo de caso em um ambiente de aprendizagem virtual que utiliza jogos para a educação empreendedora. Foi possível concluir que a utilização de gamification nesse contexto é uma alternativa a ser considerada. Observou-se que a plataforma em estudo desenvolve poucas CCEs, porém, a partir da implementação das propostas de melhoria aqui apresentada, espera-se mais engajamento dos participantes do Desafio Universitário e maior desenvolvimento das CCEs nos universitários.

As sugestões aqui apresentadas de ajustes e novas funcionalidades da plataforma poderão ser consideradas pelo SEBRAE no desenvolvimento de uma nova plataforma que, uma vez implementada, poderá ser validada empiricamente não só sua adequação aos propósitos estabelecidos, como, por meio de técnicas de meta -avaliação (Coosby e Caracelli, 2009), o próprio instrumento de avaliação aqui proposto.

\section{REFERÊNCIAS}

CHOU, Y.-K. Octalysis: complete gamification framework. 2014. <http://www. yukaichou.com/gamification-examples/octalysis-complete-gamification-framework/>.

COOSBY, L.; CARACELLI, V. Metaevaluation in practice. Journal of MultiDisciplinary Evaluation, v. 6, n. 11, 2009.

DETERDING, S. Gamification: designing for motivation. Interactions, v. 19, n. 4, p. 14-17, 2012.

DETERDING, S.; DIXON, D.; KHALED, R.; NACKE, L. From game design elements to gamefulness: defining "gamification". In: INT. ACADEMIC MINDTREK CONFERENCE. 15., 2011, Tampere, Finland. Proceedings. 2011. p. 9-15.

FURIÓ, D.; GONZÁLEZ-GANCEDO, S.; JUAN, M. C.; SEGUÍ, I.; COSTA, M. The 
efects of the size and weight of a mobile device on an educational game. Journal Computers \& Education, v. 64, p. 24-41, 2013.

GSTRAUNTHALER, T., HENDRY, S. Entrepreneurial and Accounting Education through Action-Based learning: The Genesis Project. Journal of Entrepreneurship Education, v.14, p.125-147, 2011.

HAMARI, J.; KOIVISTO, J.; SARSA, H. Does gamification work? - a literature review of empirical studies on gamification. In HAWAII INTERNATIONAL CONFERENCE ON SYSTEM SCIENCES, 4., 2014, Proceedings. p. 6-9, 2014.

HEINONEN, J., POIKKIJOKI, S. (2006). An entrepreneurial-directed approach to entrepreneurship education: mission impossible? Journal of Management Development, v. 25, n. 1, p. 80-94.

LANE, P. Innovative Teaching to Engage and Challenge Twenty-first Century Entrepreneurship Students. Journal of Entrepreneurship Education, v.14, p. 105-123, 2011.

MARCZEWSKI, A. Gamification: a simple introduction. Andrzej Marczewski, 2013. MAYER, I. et al. The research and evaluation of serious games: Toward a comprehensive methodology. British Journal of Educational Technology, 2013.

McCLELLAND, D. Characteristics of successful entrepreneurs. The Journal of Creative Behavior. v. 21, n. 3. p. 219-233, 1987.

McClELLAND, D. The achieving society. New York: VanNostrand, 1961.

NAB, J., PILOT, A., BRINKKEMPER, S., Berge, H. Ten. Authentic competence -based learning in university education in entrepreneurship. International Journal of Entrepreneurship and Small Business, v.9, n.1, p.20, 2010.

PEDERSEN, B. B. B.; POULSEN, M. L. A systematic review of gamification in education: Towards a more structured use of game mechanics. Aarhus Universitet, 2016, Dissertação de Mestrado.

SEBRAE. Desafio Universitário Empreendedor. 2015. Disponível em: <http://desafiouniversitarioempreendedor.sebrae.com.br/plataforma/index.xhtml>.

SILVA, Z. G. O perfil psicológico do empreendedor. Belo Horizonte: Manual do Modelo CEFE - GTZ/LUSO CONSULT/CENTRO CAPE, 1991.

SMALE, S. de; OVERMANS, T.; JEURING, J. GRINT, L. van der. The effect of simulations and games on learning objectives in tertiary education: a systematic review. In: Lecture Notes in Computer Science, v. 9599, p. 506-516.

VINCETT, P. S., FARLOW, S. "Start-a-Business": An Experiment in Education Through Entrepreneurship. Journal of Small Business and Enterprise Development, v. 15, n. 2, p. 274-288, 2008.

WERBACH, K.; HUNTER, D. For the win: how game thinking can revolutionize your business. Wharton Digital Press, 2012.

ZHENG, L.; YANG, L. Entrepreneurship education and employment performance: An empirical study in Chinese university. Journal of Chinese Entrepreneurship, v. 3, n. 3, p.195-203, 2011.

ZICHERMANN, G.; CUNNINGHAM, C. Gamification by design: implementing game mechanics game mechanics in Web and mobile apps. O'Reilly Media, 2011. 